\title{
Recovery of viable but non-culturable Campylobacter jejuni
}

\author{
D. M. Jones, ${ }^{*}$ E. M. SutCliffe and A. CuRry \\ Public Health Laboratory, Withington Hospital, Manchester M20 8LR, UK
}

(Received 12 February 1991; revised 7 May 1991; accepted 10 July 1991)

\begin{abstract}
Suspensions of Campylobacter jejuni became non-culturable after storage in sterilized pond water at $4^{\circ} \mathrm{C}$ for periods between 18 and $28 \mathrm{~d}$, depending on the strain. Suspensions of four strains of $C$. jejuni that had been in water for 6 weeks, and shown to be non-culturable, were fed to suckling mice. Colonization of mice was established with two of the strains and failed with the other two strains. Examination of these suspensions under the electron microscope showed some cocci having the appearance of being viable, but most cocci and all remaining spiral forms showed extensive degeneration. The results indicate that non-culturable coccal forms of $C$. jejuni are capable of infecting mice but that this property may differ between strains.
\end{abstract}

\section{Introduction}

Although the sources of human Salmonella and Campylobacter infections are broadly similar, the epidemiology of these two infections is quite different. One of the reasons for this is that salmonellas can multiply in food and the environment but campylobacters are unable to do so. It has been suggested that on prolonged exposure to water Campylobacter jejuni enters into a dormant phase during which it is no longer possible to culture the organism using conventional media, but in which viability is maintained (Rollins \& Colwell, 1986). In an outbreak of Campylobacter gastroenteritis, due to contaminated drinking water, cases were observed to occur for many days after attempts to culture $C$. jejuni from water were no longer positive, and secondary infection was discounted (Palmer et al., 1983). On a farm where the majority of the chickens were colonized by a particular serotype of $C$. jejuni that was also causing infection in the local community, treatment of the water supply on the farm resulted in the disappearance of the serotype from the chickens although campylobacters had not at any time been isolated from the chicken-shed water supply (Pearson et al., 1988). The existence of a dormant form of thermophilic campylobacters may therefore exert an important influence on concepts of the epidemiology of human and animal Campylobacter infection, and there is a need for the development of methods for the detection of non-culturable forms. In previous experiments with laboratory microcosms we observed that following prolonged exposure to water, $C$. jejuni became coccal in form and that Campylobacter antigen remained detectable for many months after the organisms were no longer culturable (Sutcliffe et al., 1991). Long after the organisms were no longer culturable on conventional media, electron microscopy demonstrated that a proportion of the cocci in the microcosms did not show signs of degeneration, consistent with continued viability. In this study we took both culturable and non-culturable preparations made from a selection of thermophilic Campylobacter strains and explored the conditions under which they colonize infant mice.

\section{Methods}

Cultures. Stock Campylobacter cultures were stored in glycerol broth at $-70^{\circ} \mathrm{C}$. C. jejuni 5813 , serotype Lior 7 Penner 4,13,50 was isolated from a patient in a milk-borne outbreak of Campylobacter infection. $C$. jejuni 9633, serotype Lior 6 Penner 6, was isolated from a patient in a water-borne outbreak of Campylobacter infection at a school (Palmer $e t$ al., 1983). C. jejuni $\mathrm{P} 10$ is a prototype strain (serotype 10) provided by Dr J. L. Penner, Toronto. $C$. jejuni 7949, serotype Lior 2 Penner 1 was isolated from a cloacal swab of a chicken on a farm where the majority of birds were colonized by this strain. C. coli 7139 , not serotypable, was isolated from a pond inhabited by moorhens and ducks.

Mice. Pregnant BALB/c mice were obtained from Bantin and Kingman Ltd, and newborn mice were fed with $0.02 \mathrm{ml}$ bacterial suspension on the first day after birth.

Preparation of cultures for feeding. Campylobacter cultures were grown on horse blood agar for $48 \mathrm{~h}$ at $37^{\circ} \mathrm{C}$ in a microaerobic atmosphere achieved with an Anaerobic Gas Generating Kit (Oxoid) used without a catalyst. The culture was suspended in nutrient broth and dilutions made in $4 \%(w / v)$ skimmed cows' milk (Marvel) in sterile distilled water. Dilutions expected to contain from 10 to $10^{4}$ c.f.u. per $0.02 \mathrm{ml}$ were fed to mice on various occasions, and surface viable counts were made simultaneously on these suspensions to establish the precise number of organisms given. In addition to using the strains from frozen storage, strains 5813 and 7949 were also used as a challenge after a single passage in mice and a single subculture from $-70^{\circ} \mathrm{C}$. When it 
had been established that only small numbers of organisms were required for colonization, on one occasion only two infants in a litter were fed the colonizing dose of strain 5813 and the whole litter was subsequently examined for colonization.

Preparation of microcosms. Water for the establishment of microcosms was collected from a pond that was known to sustain the presence of Campylobacter spp., filtered through a Whatman general purpose filter and sterilized at $121^{\circ} \mathrm{C}$ for $15 \mathrm{~min}$. Campylobacter strains were grown on blood agar at $37^{\circ} \mathrm{C}$ for $48 \mathrm{~h}$ in a microaerobic atmosphere. The entire growth from one plate was suspended in $100 \mathrm{ml}$ sterile pond water and stored at $4{ }^{\circ} \mathrm{C}$ without agitation in the dark. When prepared in this way the initial cell count was approximately $10^{7}$ c.f.u. $\mathrm{ml}^{-1}$.

Examination of suspensions for culturability. Methods used were as follows: (1) direct culture on $10 \%(\mathrm{v} / \mathrm{v})$ horse blood agar or $10 \%(\mathrm{v} / \mathrm{v})$ sheep blood agar (Oxoid BA base no. 2); (2) filtration of $10 \mathrm{ml}$ suspension through a $0.45 \mu \mathrm{m}$ filter which was then placed on blood agar for $24 \mathrm{~h}$ before removal and further incubation of the plate (Pokorny, 1990); (3) inoculation of $5 \mathrm{ml}$ suspension into $5 \mathrm{ml}$ Brucella broth (Oxoid); (4) pre-enrichment of $5 \mathrm{ml}$ suspension in $5 \mathrm{ml}$ Ringer's solution before direct culture on blood agar and enrichment culture in Preston Campylobacter Enrichment Broth (Oxoid); (5) mixture of $5 \mathrm{ml}$ suspension with $5 \mathrm{ml}$ double-strength Enrichment Broth with or without antibiotics, incubated at $37^{\circ} \mathrm{C}$ and subcultured on to blood agar at $48 \mathrm{~h}$ intervals for 2 weeks.

Feeding of non-culturable suspensions. After 6 weeks at $4^{\circ} \mathrm{C}, 10 \mathrm{ml}$ of the Campylobacter suspension was removed from each microcosm and centrifuged at 3000 r.p.m. for $30 \mathrm{~min}$. The deposit was resuspended in $0.5 \mathrm{ml} 4 \%$ skimmed milk. Each infant mouse in the litter was fed $0.02 \mathrm{ml}$ of this suspension with a plastic pipette. Seven days later the litter and the mother were killed and approximately $1 \mathrm{~cm}$ of lower intestine from each was placed in the enrichment medium which was incubated at $37^{\circ} \mathrm{C}$ for up to 2 weeks with subculture every $2 \mathrm{~d}$ on to selective medium [Campylobacter Blood-Free selective agar with cefoperazone (Oxoid)].

After recovery of strains from mice, and from prolonged periods in microcosms, all strains of Campylobacter sp. were reserotyped to establish identity with the original strain.

Electron microscopy. Non-culturable suspensions were negatively stained and examined by transmission electron microscopy.

\section{Results}

\section{Colonization by viable organisms}

Infant mice were asymptomatically colonized after feeding with doses of $10^{3}$ and $10^{2}$ c.f.u. of strain 5813, but 10 c.f.u. failed to colonize. When two infant mice of a litter of six were fed 50 c.f.u. of strain 5813 , the whole litter was colonized one week later. C. jejuni 7949 colonized mice following a dose of $10^{3}$ c.f.u. but the minimal colonizing dose was not established. $C$. jejuni P10 colonized the mice after feeding with $6 \times 10^{4}$ c.f.u. but there was no colonization following feeding with $6 \times$ $10^{2}$ c.f.u. Mice were not colonized after feeding with $2 \times$ $10^{6}$ c.f.u. of $C$. coli 7139 ; the colonizing dose of strain 9633 was not investigated.
Table 1. Colonization of suckling mice fed with nonculturable $C$. jejuni stored in water at $4^{\circ} \mathrm{C}$ (unless otherwise stated) for 6 weeks

Mice in one litter were fed $0.02 \mathrm{ml}$ suspension of the indicated strain of $C$. jejuni on the first day of life.

\begin{tabular}{lc}
\hline \hline \multicolumn{1}{c}{ Strain } & $\begin{array}{c}\text { No. colonized/ } \\
\text { no. inoculated }\end{array}$ \\
\hline 5813 & $2 / 6$ \\
5813 passaged & $1 / 5$ \\
5813 held at $21^{\circ} \mathrm{C}$ & $0 / 11$ \\
9633 & $0 / 6$ \\
P10 & $0 / 5$ \\
7949 & $0 / 5$ \\
7949 passaged & $3 / 5^{*}$ \\
\hline \hline
\end{tabular}

* With secondary spread to parent.

\section{Duration of culturability when stored in water}

The most sensitive of culture methods used was to add $5 \mathrm{ml}$ suspension to double-strength enrichment broth, incubate at $37^{\circ} \mathrm{C}$, and subculture at $48 \mathrm{~h}$ intervals for 2 weeks. With this method it was possible to recover campylobacters from stored microcosms for up to $3 \mathrm{~d}$ after other methods had become negative. While the enrichment broths were subcultured repeatedly for up to 2 weeks, most of those that were positive yielded subcultures within $48 \mathrm{~h}$ and no extra positive results were obtained beyond $6 \mathrm{~d}$ incubation. In microcosms at $4{ }^{\circ} \mathrm{C}$, strain 5813 could be cultured for up to $28 \mathrm{~d}, 7139$ was culturable for up to $21 \mathrm{~d}$, and 7949 remained culturable for $18 \mathrm{~d}$. In a microcosm at $21^{\circ} \mathrm{C}$, strain 5813 remained culturable for 3 weeks. The precise period before other strains became non-culturable was not ascertained. As none of the five strains in the study could be cultured after 4 weeks and the protocol required 2 weeks to establish this, all mice were fed with non-culturable suspensions that had been held for a minimum of 6 weeks. In one experiment, strain 5813 was used after storage in water at $21^{\circ} \mathrm{C}$ for 6 weeks.

\section{Electron microscopy}

The non-culturable suspensions contained bacterial debris among which were cells with spiral morphology. All of these showed evidence of extreme protoplast shrinkage away from the outer envelope, particularly at the poles, and the envelope appeared creased and penetrated by stain. The flagellar filaments were commonly represented by a shortened stump. About $20 \%$ of the bacterial cells were spherical in form and most of these also showed protoplast shrinkage, stain penetration and fragmentation of flagellar filaments (Fig. 1). About a third of the spherical cells had a smooth 


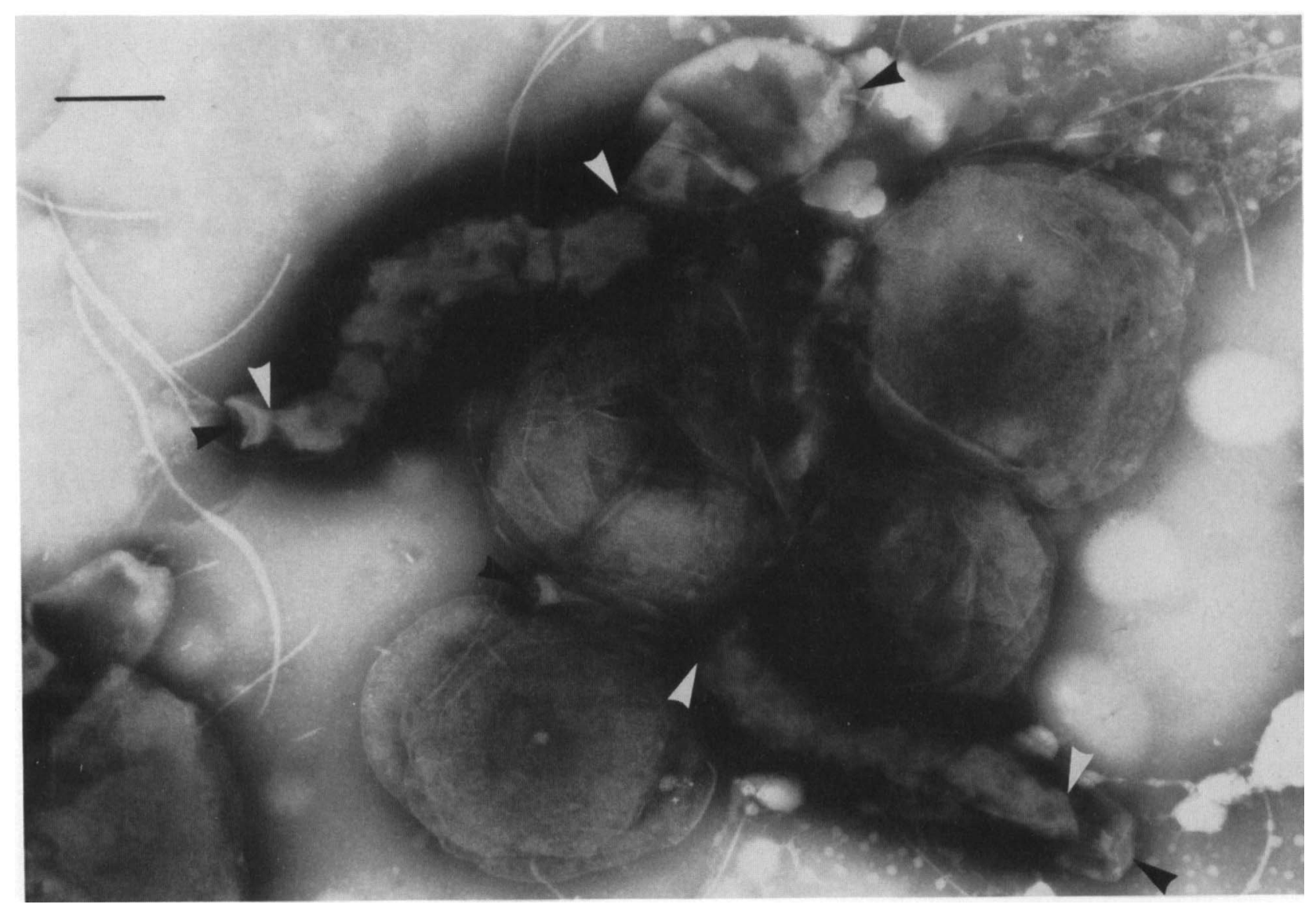

Fig. 1. Negatively stained non-culturable suspension of $C$. jejuni showing organisms with spiral and coccal morphology. The spiral forms show protoplast shrinkage and extensive surface creasing. Black arrowheads indicate the original poles of the organism; white arrowheads indicate the extremities of the shrunken protoplast. The coccal forms in this field also show surface creasing indicative of degenerative change. Bar, $300 \mathrm{~nm}$.

intact cell wall (approximately $6 \%$ of the total number of cells) and no evidence of protoplast shrinkage or stain penetration (Figs 2 and 3). These cells were mostly biflagellate; each flagellum was of normal length and originated from a circular concavity, the flagellar insertions being separated by only a short distance on one side of the coccus. The flagellar filaments of these viable-looking cells showed no sign of fragmentation. The absence of any degenerative features indicates possible viability of some of the coccal forms.

\section{Colonization from non-culturable suspensions}

Under the conditions of these experiments, colonization of mice occurred with strains 5813 and 7949, but was not achieved with 9633 and P10 (Table 1). Because large numbers of culturable cells of strain 7139 failed to colonize, this strain was not investigated with nonculturable suspensions.

\section{Discussion}

Infant mice on the first day of life could be colonized by feeding with culturable suspensions of $C$. jejuni, but there was variation in colonizing ability between the strains chosen for study. Strains also varied in the time taken to become non-culturable when stored in water. $C$. jejuni 5813 was the most durable strain (4 weeks); 7139 and 7949 both remained culturable for about 3 weeks at $4{ }^{\circ} \mathrm{C}$. We had previously noted that storage of microcosms at higher temperatures shortened the period of culturability (Sutcliffe et al., 1991) and 5813 remained culturable for only 3 weeks at $21^{\circ} \mathrm{C}$. There is an interesting parallel between these results and a study of the survival of campylobacters in sewage. It was found that 22-d-old digested sewage only yielded positive Campylobacter cultures in the coldest months of the year, despite being present in raw sewage throughout the year (Jones et al., 1990). It is reasonable to assume that the periods of both 


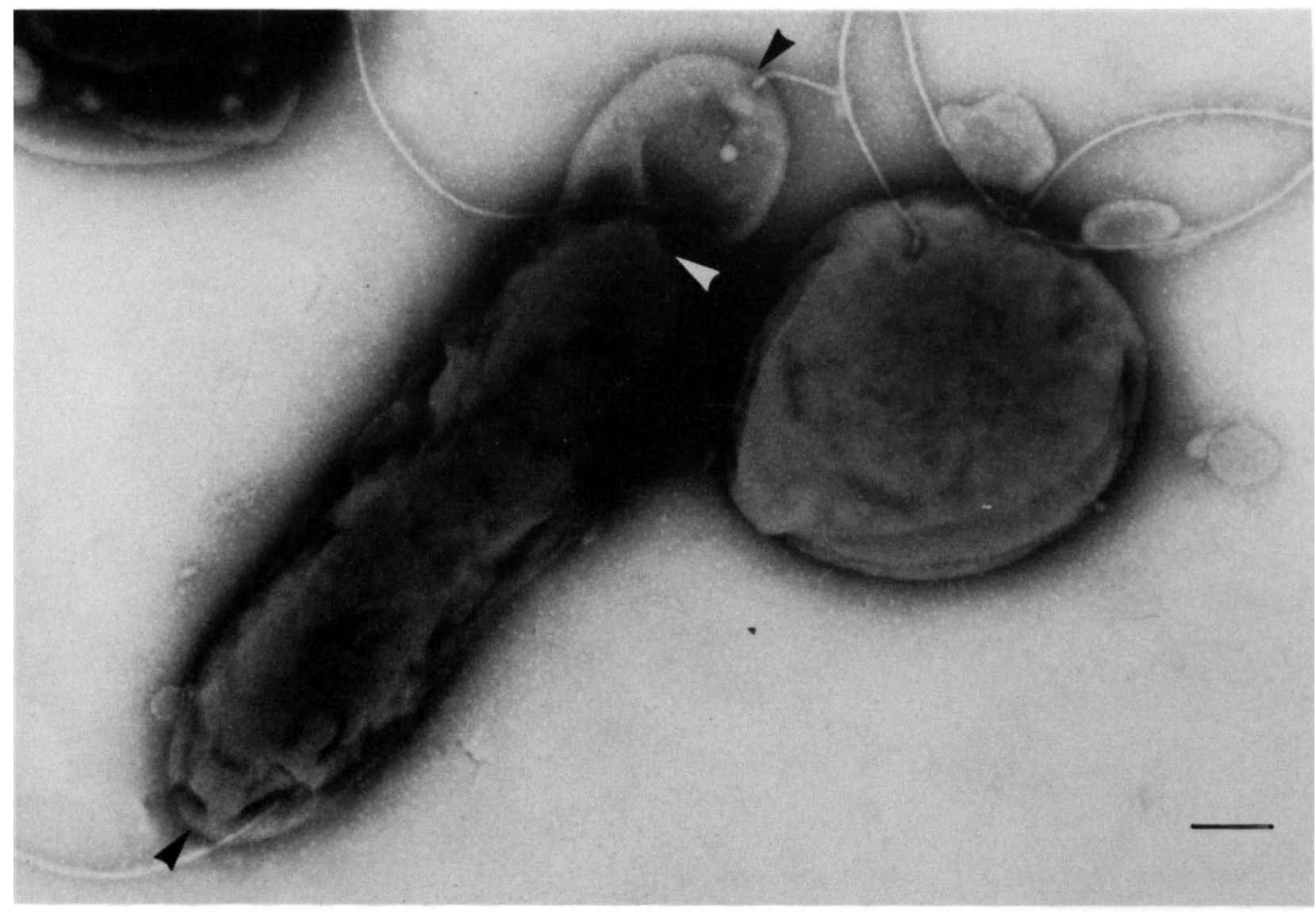

Fig. 2. Negatively stained non-culturable $C$. jejuni. The spiral organism shows evidence of protoplast shrinkage, surface creasing and flagellar fragmentation. Black arrowheads indicate the poles of the organism; the white arrowhead shows the point to which protoplast shrinkage has occurred. The coccus shows little evidence of surface creasing or protoplast shrinkage and there are two intact flagellar filaments originating from surface pits. This coccus has none of the degenerative changes seen in the majority of organisms. Bar, $150 \mathrm{~nm}$.

culturability and viability will be shortened at temperatures above $4{ }^{\circ} \mathrm{C}$ and this has been observed using other in vitro methods to indicate viability (Rollins \& Colwell, 1986).

The definition of non-culturability clearly depends on the efficiency of the media at recovering damaged or dormant organisms. We used a variety of conventional media, various temperatures and avoided selective agents, but in our microcosms culturability by these methods was lost after 3 to 4 weeks at $4{ }^{\circ} \mathrm{C}$. Storing the suspensions for a minimum of 2 weeks after loss of culturability before feeding to mice allowed a margin to ensure that the persistence of any culturable cells was unlikely. Under the electron microscope all the spiral forms in these suspensions appeared degenerate, and their structure so distorted that it is difficult to believe they could be viable. Most of the coccal forms also showed degenerative changes but a small proportion did retain structural integrity and had the appearance we associate with viability. $C$. jejuni 5813 proved to be the most able to colonize mice from the 6 week nonculturable suspensions. Culturable organisms, indistinguishable from the original strain, were isolated from two of a litter of six mice 1 week after feeding with the non-culturable suspension. This implies that at least two infective doses were present in the six $0.02 \mathrm{ml}$ amounts of the 6-week-old suspension fed to the litter. A repeat experiment with this strain after mouse passage gave a similar result. Examination of the suckling mice 1 week after feeding with a non-culturable suspension appeared to provide insufficient time for multiplication and then excretion to infect other infant mice in the litter. In contrast, feeding two out of six mice with 50 culturable organisms of strain 5813 produced colonization of the whole litter after 1 week. This may indicate that a finite time is necessary for the metabolic processes associated 


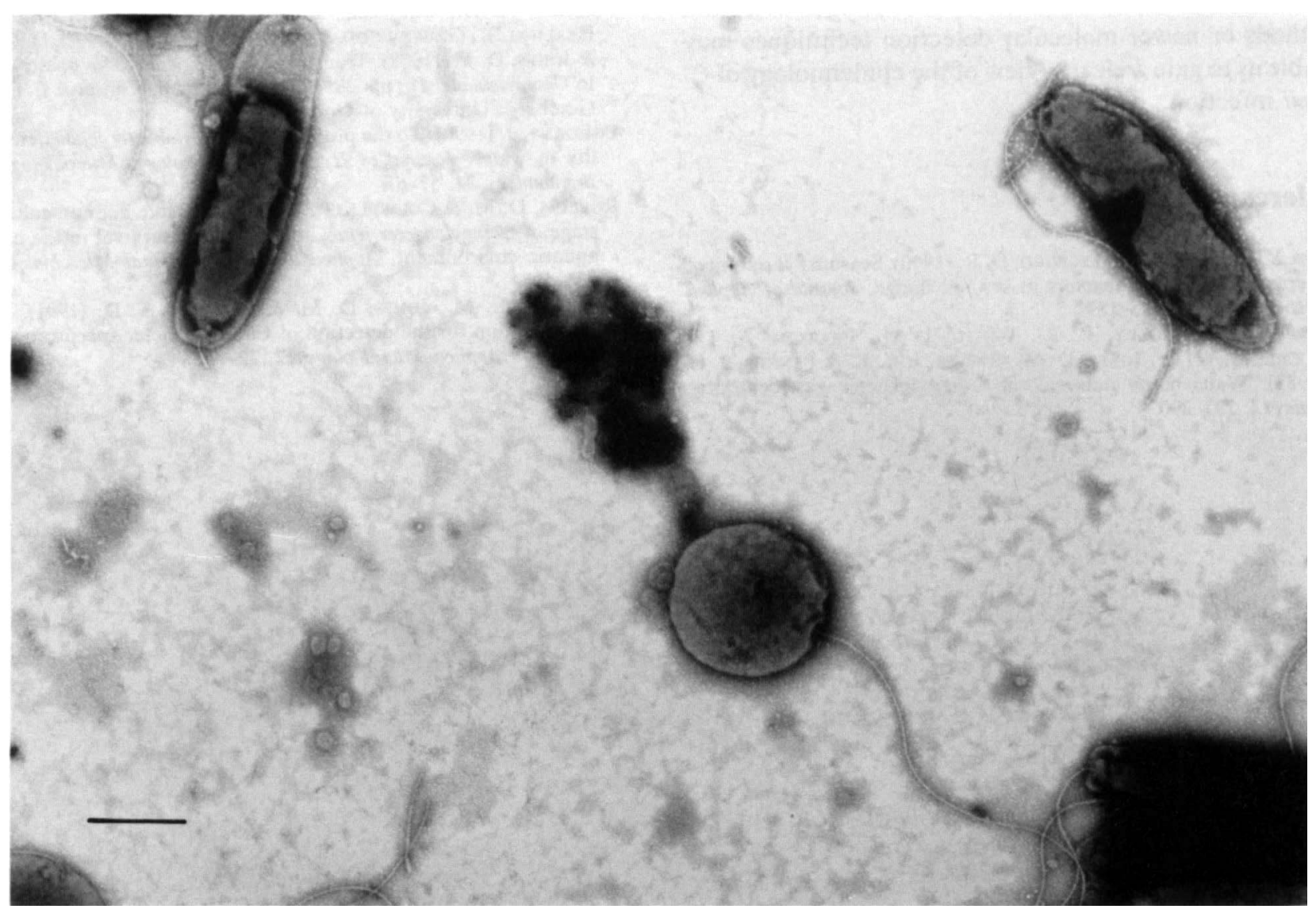

Fig. 3. Negatively stained suspension of $C$. jejuni after 6 weeks in water. Rods show cytoplasmic shrinkage and flagellar fragmentation; the coccus $(0.75 \mu \mathrm{m}$ diam.) shows no sign of cytoplasmic shrinkage, has an intact flagellum and the appearance associated with viability. Bar, $500 \mathrm{~nm}$.

with replication to be 'switched on' again in the viable/non-culturable organism and so the process of colonization and excretion takes longer than with culturable organisms.

Mouse passage of $C$. jejuni may enhance the ability to colonize mice even in the non-culturable form, as a first experiment of feeding with a non-culturable suspension of strain 7949 failed to induce colonization, but a similar suspension made after mouse passage did so. Nonculturable suspensions of $C$. jejuni 9633 and P10 failed to colonize mice in these studies. This indicated that either their lower colonizing ability in the culturable state extended to the non-culturable state or that they had progressed to non-viability within the 6 week storage period.

These findings may have some relevance to the spread of $C$. jejuni from water or other environmental sources in commercial chicken-rearing houses. Although nonculturable suspensions colonize mice, the susceptibility of the mouse to colonization is likely to be less than the natural avian host. It is likely that ingestion of even smaller numbers of non-culturable cells can initiate colonization in young chickens and that secondary infection then results in the high proportion of colonized birds seen in broiler flocks.

The animal gut remains the only natural site of multiplication for thermophilic campylobacters, but the organism has a durable dormant form which enables it to survive in the environment, particularly at low temperatures. In progression to this form, the cells become coccal and a resting metabolism ensues for a period before complete loss of viability. These experiments show that at some stage after loss of culturability the cells are still able to come out of dormancy in the animal gut and begin to replicate. These findings are consistent with those epidemiological situations where water-borne transmission of $C$. jejuni is implicated but culture techniques fail to confirm this. The techniques used in this study are unsuitable for epidemiological studies but appreciation of the existence of the viable but non-culturable state 
taken in conjunction with the results of antigen detection methods or newer molecular detection techniques may enable us to gain a clearer view of the epidemiology of $C$. jejuni infection.

\section{References}

Jones, K., Betaieb, M. \& Telford, D. R. (1990). Seasonal variation of thermophilic campylobacters in sewage sludge. Journal of Applied Microbiology 69, 185-189.

Palmer, S. R., Gully, P. R., White, J. M., Pearson, A. D., Suckling, W. G., Jones, D. M., Rawes, J. C. L. \& Penner, J. L. (1983). Water-borne outbreak of Campylobacter gastroenteritis. Lancet i, 287-290.
Pearson, A. D., Colwell, R., Rollins, D., Watkin-Jones, M., Healing, T., Greenwood, M., Shahamat, M., Jump, E., Hood, M. \& JONES, D. M. (1988). Transmission of $C$. jejuni on a poultry farm. In Campylobacter IV, pp. 281-284. Edited by B. Kaijser \& E. Falsen. Goteberg: University of Goteberg.

POKORNY, J. (1990). To the problem of Campylobacter jejuni detectability in water. Journal of Hygiene, Epidemiology, Microbiology and Immunology 34, 57-61.

Rollins, D. M. \& Colwell, R. R. (1986). Viable but non-culturable stage of Campylobacter jejuni and its role in survival in the natural aquatic environment. Applied and Environmental Microbiology 52, 531-538.

Sutcliffe, E. M., Jones, D. M. \& Pearson, A. D. (1991). Latex agglutination for the detection of Campylobacter species in water. Letters in Applied Microbiology 12, 72-74. 